\title{
Diagnostic capacity of SD-OCT segmented ganglion cell complex versus retinal nerve fiber layer analysis for congenital glaucoma
}

\author{
Laura Morales-Fernandez ${ }^{1}$ Maria Jimenez-Santos ${ }^{1} \cdot$ Jose M. Martinez-de-la-Casa $^{2}$ Ruben Sanchez-Jean $\mathbb{D I}^{2}$. \\ Maria Nieves $^{1}$ - Federico Saenz-Frances ${ }^{1} \cdot$ Sofia Garcia-Saenz ${ }^{1} \cdot$ Lucia Perucho $^{1} \cdot$ Rosario Gomez-de-Liaño $^{1}$. \\ Julian Garcia-Feijoo $2,3,4$
}

Received: 8 August 2017 / Revised: 15 November 2017 / Accepted: 8 January 2018 / Published online: 12 April 2018

(c) The Royal College of Ophthalmologists 2018

\begin{abstract}
Purpose To compare the diagnostic performance of circumpapillary retinal nerve fiber layer (cpRNFL) analysis versus segmented ganglion cell complex analysis both by spectral-domain optical coherence tomography (SD-OCT) in children with primary congenital glaucoma (PCG).

Methods Participants were 40 children diagnosed with PCG and 60 healthy children. Ophthalmological data collected (for one eye per child) were cup-disc ratio (C/D) and axial length (AL). SD-OCT with automated segmentation was used to measure the thicknesses and volumes of the macular retinal nerve fiber layer (mRNFL), ganglion cell layer (GCL), and inner plexiform layer (IPL). For the cpRNFL measurements conventional S-D OCT software was used and the capacity of each method to discriminate between normal and glaucomatous eyes was compared.

Results Mean age was $11.20 \pm 3.94$ years for the glaucoma patients and $10.90 \pm 2.46$ years for controls $(p=0.64)$. All measurements were reduced (thinner) in the glaucoma group, significantly so for: cpRNFL, GCL, IPL and outer-superior and outer-inferior quadrant mRNFL. According to their areas under the receiver operating characteristics curve (AUC), temporal superior cpRNFL (0.869) and outer superior GCL (0.840), IPL (0.799), and mRNFL (0.767) showed the better diagnostic capacity. No differences were observed in AUCs for the most discriminatory cpRNFL and macular measurements.

Conclusion Segmented macular layer analysis shows a good capacity to discriminate between normal and glaucomatous eyes; which is comparable to that of cpRNFL analysis in children with PCG.
\end{abstract}

$\triangle$ Laura Morales-Fernandez

lauramoralesfernandez@gmail.com

1 Ophthalmology Unit, Hospital Clinico San Carlos, Instituto de Investigacion Sanitaria del Hospital Clinico San Carlos (IdISSC), Madrid, Spain

2 Ophthalmology Unit, Hospital Clinico San Carlos, Deptartment of Ophthalmology and ORL, Faculty of Medicine, Universidad Complutense de Madrid, Instituto de Investigacion Sanitaria del Hospital Clinico San Carlos (IdISSC), Madrid, Spain

3 Instituto de Investigaciones Oftalmologicas Ramon Castroviejo, Universidad Complutense de Madrid, Madrid, Spain

4 Cooperative Research Network on Age-Related Ocular Disease, and Visual and Life Quality, Instituto de Salud Carlos III, Madrid, Spain

\section{Introduction}

Primary congenital glaucoma (PCG) is a common form of glaucoma in childhood, causing significant vision loss in affected children. The standardized definition of PCG according to the Childhood Glaucoma Research Network (CGRN) classification determined in the 9th World Glaucoma Association Consensus meeting [1] is based on clinical signs, that includes children had IOP $>22 \mathrm{mmHg}$ at diagnosis, with clinical evidence of glaucoma such as enlarged corneal diameter, Haab's striae and glaucomatous optic disc head appearance.

Although PCG diagnosis is based on a clinical examination [1], the application of new structural diagnostic devices used in adults such as Optic Coherence Tomography (OCT) might enable clinicians to obtain valuable objective measurements [2,3].

OCT is a non invasive transpupilar imaging method that produces high-resolution, cross-sectional tomographic 
images of retinal tissue that correlate well with retinal histology [4]. The development of Spectral Domain OCT (SDOCT) allows a quicker acquisition of higher quality images than time domain OCT [5]. These advantages offered by SD-OCT provide clear benefits for the management of pediatric glaucoma in the clinical practice.

SD-OCT has become an essential tool for adult glaucoma management. Firstly, Analysis of the circumperipapillary retinal nerve fiber layer (cpRNFL) showed an excellent reproducibility and a high diagnostic ability for glaucoma in adults [6]. And secondly, the thickness measurement of the ganglion cell complex (GCC) with SD-OCT devices have shown very good discriminative diagnostic ability, particularly in adults with early glaucoma, which is comparable to that obtained with cpRNFL analysis [3, 7].

The latest version of the Spectralis SD-OCT software (Heidelberg Engineering GmbH, Heidelberg, Germany) allows for automatic individual segmentation of the three inner retinal layers most affected by glaucoma: the macular retinal nerve fiber layer (mRNFL), ganglion cell layer (GCL), and inner plexiform layer (IPL) [8].

In pediatric glaucoma, a decreased of cpRNFL thickness compared to healthy children and good reproducibility of these measurements were observed using several SD-OCT devices [9, 10], including Spectralis OCT [3]. On the contrary, there is no available information about the utility of the Spectralis OCT segmentation software of inner macular layers for pediatric glaucoma.

This study was designed to assess the diagnostic capacity for PCG in children of Spectralis SD-OCT measurements made of the inner macula layers using this new segmentation software versus cpRNFL measurements made using conventional analysis.

\section{Materials and methods}

\section{Study participants}

This was an observational cross-sectional study in which we examined 60 eyes of 60 healthy children and 40 eyes of 40 age-matched Caucasian children with PCG. Participants were aged 4 to 16 years. The study protocol adhered to the tenets of the Declaration of Helsinki and was approved by the Ethics Committee of the Hospital Clínico San Carlos (HCSC), Madrid, Spain. Written informed consent was obtained from the parents or legal guardian of each child, and each child gave their verbal consent to participate in our study.

All participants underwent a comprehensive ophthalmologic examination by a single examiner (LMF) in which the variables recorded were visual acuity and refractive state,dilated fundus examination, cup-disc ratio (C:D), and axial length using a Lenstar LS-900 ocular biometer (Haag Streit AG, Koeniz Switzerland). To minimize the effect of refractive error on SD-OCT testing, eyes with a spherical equivalent greater than \pm 6 diopters were excluded.

As the control group, 60 healthy children were recruited among those undergoing a routine ophthalmologic exam, the children of adult patients, and children of staff members who agreed to participate in this study. Eyes were defined as normal (in the absence of other features of glaucoma) if they showed a visual acuity of $\geq 0,8$ (Snellen Scale), an optic nerve cup-disc asymmetry of $\leq 0.2$, and a cup-disc ratio of $<0.5$ without focal neuroretinal rim loss, hemorrhage, or pallor. Children were excluded if they had a family history of glaucoma or had been diagnosed with amblyopia or strabismus. All measurements were made in both eyes, but the only the data of one randomly selected eye were entered in the analysis.

For the patient group, we recruited 40 children who had been diagnosed with PCG and were managed at the Dept. of Glaucoma of the HCSC. All patients fulfilled the diagnostic criteria for PCG according to the CGRN classification: [1] IOP $>22 \mathrm{mmHg}$ upon diagnosis, clinical evidence of glaucoma such as enlarged corneal diameter, Haab's striae and glaucomatous appearance of the optic nerve head. Eyes were included if they had sufficient visual acuity for fixation, equal or higher than 0.1 using Snellen Scale. When both eyes were eligible, one eye was randomly selected using a randomization list obtained from www.randomization.com.

\section{Optical coherence tomography}

The same instrument Spectralis OCT (software version 6.3.2.0) (Heidelberg Engineering GmbH, Heidelberg, Germany) was used for cpRNFL thickness mapping and the macula examination.

All scans were performed by the same experienced operator (RSJ). Macular and circumpapillary scans were performed with pupil dilation in a dark room on the same day as the other tests. This instrument's automatic eye tracking technology (TruTrack; Heidelberg Engineering) maintains fixation on the retina. Only well-centered images with quality $(\mathrm{Q})$ of $>20 \mathrm{db}$ provided by the instrument were accepted.

\section{Circumpapillary RNFL thickness mapping}

For SD-OCT RNFL thickness measurements, circular scans were conducted with a scanning angle of 12 degrees. This assumes a standard corneal curvature of $7.7 \mathrm{~mm}$ thus projecting a circle of diameter $3.6 \mathrm{~mm}$ on the retina. The Spectralis device's software divides a full circumpapillary circle scan into 6 regions and then provides: temporal quadrant thickness (90-degrees), superotemporal sector 
a
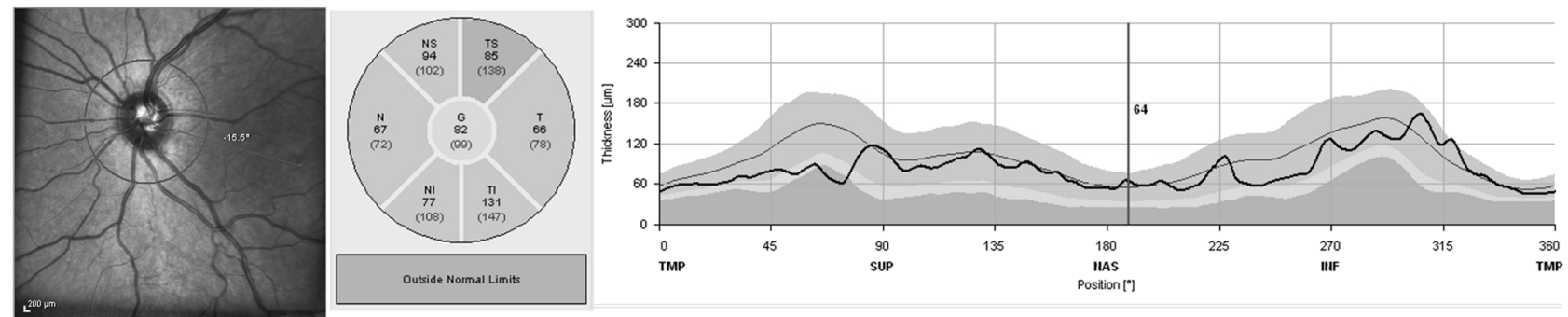

b

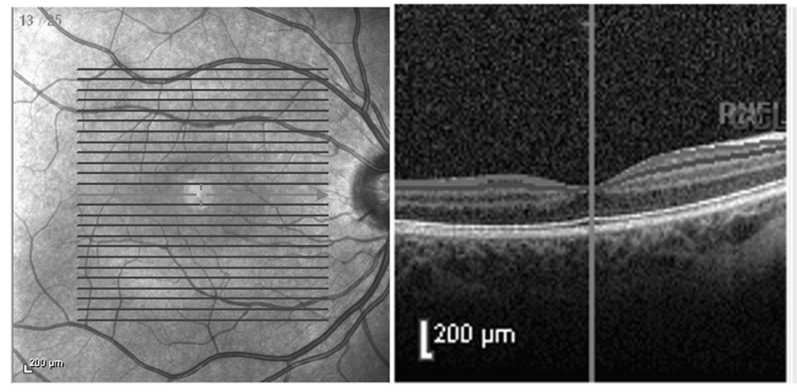

Fig. 1 Representative Spectralis OCT scans of cpRNFL thickness (a) and macular thickness scan (b). The Spectralis device's software divides a full circumpapillary circle scan into 6 regions and macular

thickness (45-degrees), superonasal sector thickness (45degrees), nasal quadrant thickness (90-degrees), inferonasal sector thickness (45-degree sector), inferotemporal sector thickness (45-degrees), and average thickness (average of all 6 sectors or 360 degrees). The latter is provided as a number in the center (Fig. 1).

\section{Inner macular layer thicknesses}

For SD-OCT inner macular layer segmentation, a scanning area of $5,8 \mathrm{~mm} \times 5,8 \mathrm{~mm}$ centered at the fovea was examined with fast macular cube, containing 25 B-scans, separated by 242 microns. For thickness measurements, macular and inner retinal layers were defined as in the Early Treatment of Diabetic Retinopathy Study (ETDRS) map of the macula: a $1 \mathrm{~mm}$ ring centered at the macula was taken as the fovea, and $3 \mathrm{~mm}$ and $6 \mathrm{~mm}$ rings as inner and outer rings each divided into four zones designated superior, nasal, inferior, and temporal, were used in our analysis. The Spectralis segmentation software (version 6.3.2.0) was then used to obtain the numerical values for each of these nine zones and macular volume of the three retinal layers including retinal nerve fiber layer (mRNFL), ganglion cell layer (GCL) and inner plexiform layer (IPL) (Fig. 1).

\section{Statistical analysis}

Statistical analyses were performed using SPSS software (version 20.0; SPSS, Inc., Chicago, IL, USA). Sex and

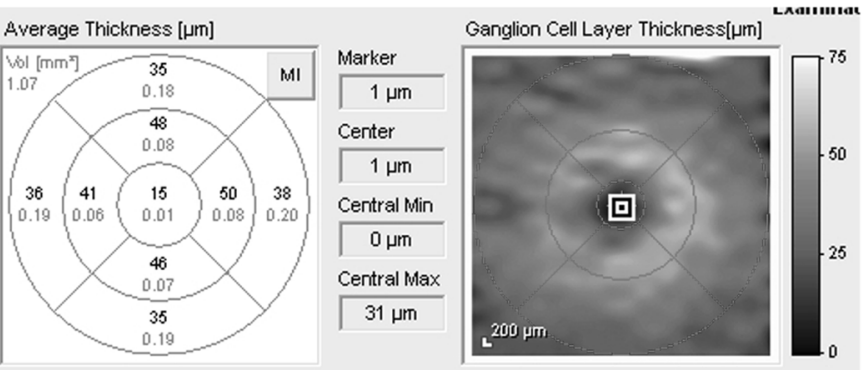

map into 9 sectors defined by an Early Treatment Diabetic Retinopathy Study (ETDRS) circle for every layer. In the figure (b), an example of the GCL layer measurements of pediatric glaucoma eye is shown

laterality differences between the normal and glaucoma groups were compared using the Pearson $X^{2}$ test. The Student's $t$ test was used to compare age, AL and C:D, the thicknesses cpRNFL, mRNFL, GCL, and IPL and macular volume between the two groups. Receiver operating characteristics curves (ROC) were constructed and areas under the curve (AUCs) used to assess the capacity of each variable to distinguish between glaucomatous and healthy eyes. AUCs were compared for the different variables using DeLong Method. Significance was set at $P$ lesser than 0.05 .

\section{Results}

The baseline demographics of the two study groups are provided in Table 1. In 13 of the 40 glaucomatous patients in which both eyes were elegibles, only one eye was randomly selected. There was no significant difference in mean age between the control subjects $(10.90 \pm 2.46$ years $)$ and glaucoma patients $(11.20 \pm 3.94$ years $)(p=0.64)$. As expected, we detected statistical differences in C:D ratio $(<0.001)$ and axial length $(<0.001)$ between the groups (Table 1).

All circumpapillary and macular thicknesses measured by OCT were reduced in the glaucoma group compared to the control group (Table 2). Differences were significant for all cpRNFL thicknesses, while for the macular analysis differences were significant for the layers GCL and 
Table 1 Demographics and ocular characteristics of the study participants

\begin{tabular}{llll}
\hline & $\begin{array}{l}\text { Control group } \\
N=60 \text { eyes }\end{array}$ & $\begin{array}{l}\text { PCG group, } \\
N=40 \text { eyes }\end{array}$ & $P$ value \\
\hline Sex, male:female & $24: 36$ & $24: 16$ & $0.16^{\mathrm{a}}$ \\
Laterality, right:left & $30: 30$ & $23: 17$ & $0.79^{\mathrm{a}}$ \\
$\begin{array}{l}\text { Age (years) Mean } \\
\text { (SD) }\end{array}$ & $10.90(2.46)$ & $11.20(3.94)$ & $0.64^{\mathrm{b}}$ \\
$\begin{array}{l}\text { Axial length (mm) } \\
\text { Mean (SD) }\end{array}$ & $23.41(1.04)$ & $24.85(1.96)$ & $<0.001^{\mathrm{b}}$ \\
C:D Mean (SD) & $0.24(0.14)$ & $0.52(0.29)$ & $<0.001^{\mathrm{b}}$ \\
\hline
\end{tabular}

C:D Cup-to-disc ratio

a Pearson $X^{2}$ test

${ }^{\mathrm{b}} t$-test

IPL. In contrast, significant differences in mRNFL thicknesses were only detected for the four outer ring quadrants. Overall volumes of each of the inner layers of the macula were lower in the glaucoma group and differences were significant for the three layers examined. No significant differences emerged between groups in thicknesses at the foveal level of any of the layers (Table 2).

In terms of distinguishing between normal and glaucomatous eyes, best AUCs were observed for the superior temporal sector $(\mathrm{AUC}=0.869)$ and overall thickness of the cpRNFL $(\mathrm{AUC}=0.835)$ and of the sectors outersuperior of the mRNFL $(A U C=0.767)$, GCL $(A U C=$ $0.840)$ and IPL $(A U C=0.799)$ (Table 3$)$. No differences were noted when we compared AUCs for the most discriminatory papillary variable, the cpRNFL superior temporal sector, and the best macular variables (Fig. 2), the outer-superior mRNFL sector $(p=0.130)$, outersuperior CCG sector $(p=0.630)$ and outer-superior IPL sector $(p=0.764)$.

\section{Discussion}

Currently, the diagnosis of PCG is based on clinical criteria and glaucomatous optic nerve damage detected by an expert remains the gold standard. Besides this, SD-OCT has proved to be useful for the management of pediatric glaucoma [11] as it provides objective cpRNFL thickness measurements in children. Reproducibility of cpRNFL measurements using Spectralis OCT has been reported to be similar in children and in adults, being excellent in both cases [3].

The recent introduction of Spectralis SD-OCT software designed for the analysis of segmented inner macular layers has rekindled interest in the management of glaucoma in adult patients. Recent studies have shown a good
Table 2 Thicknesses (microns) and volumes $\left(\mathrm{mm}^{3}\right)$ of the circumpapillary retinal nerve fiber layer, macular retinal nerve fiber layer, ganglion cell layer and inner plexiform layer in both study groups

\begin{tabular}{|c|c|c|c|}
\hline Layer measured & $\begin{array}{l}\text { Control group } \\
N=60 \text { eyes } \\
\text { Mean (SD) }\end{array}$ & $\begin{array}{l}\text { PCG group } \\
N=40 \text { eyes } \\
\text { Mean (SD) }\end{array}$ & $P$ value \\
\hline \multicolumn{4}{|l|}{ cpRNFL } \\
\hline Overall & $101.831(9.89)$ & $74.32(26.25)$ & $<0.001$ \\
\hline Sup-temporal & $143.66(18.79)$ & $94.55(39.91)$ & $<0.001$ \\
\hline Temporal & $72.94(10.63)$ & $58.72(10.63)$ & $<0.001$ \\
\hline Inf- temporal & $143.59(21.26)$ & $98.35(46.51)$ & $<0.001$ \\
\hline Inf-nasal & $118.96(27.77)$ & $81.92(35.75)$ & $<0.001$ \\
\hline Nasal & 74.37 (14.49) & $58.97(22.16)$ & $<0.001$ \\
\hline Sup-nasal & $113.67(24.85)$ & $80.72(37.10)$ & $<0.001$ \\
\hline \multicolumn{4}{|l|}{ mRNFL } \\
\hline Outer-superior & $36.81(5.33)$ & $29.02(10.73)$ & $<0.001$ \\
\hline Inner-superior & $23.16(3.17)$ & $22.65(4.78)$ & 0.522 \\
\hline Outer-temporal & $19.06(4.49)$ & $17.80(1.96)$ & 0.057 \\
\hline Inner-temporal & $16.55(1.26)$ & $17.07(1.60)$ & 0.085 \\
\hline Outer-inferior & 41.47 (15.57) & $32.19(12.62)$ & 0.001 \\
\hline Inner-inferior & $26.03(13.25)$ & $22.36(5.07)$ & 0.055 \\
\hline Outer-nasal & $50.85(9.90)$ & $42.51(14.70)$ & 0.002 \\
\hline Inner-nasal & $20.58(2.74)$ & $20.53(3.31)$ & 0.941 \\
\hline Foveal & 12.13 & $12.12(2.94)$ & 0.980 \\
\hline Volume & $0.92(0.18)$ & $0.78(0.22)$ & 0.004 \\
\hline \multicolumn{4}{|l|}{ GCL } \\
\hline Outer-superior & $37.03(3.58)$ & $29.12(6.69)$ & $<0.001$ \\
\hline Inner-superior & $53.90(4.04)$ & $44.82(12.63)$ & $<0.001$ \\
\hline Outer-temporal & $37.66(4.85)$ & $29.90(8.46)$ & $<0.001$ \\
\hline Inner-temporal & $47.93(5.02)$ & $39.46(13.27)$ & $<0.001$ \\
\hline Outer- inferior & $36.20(3.73)$ & $29.19(6.46)$ & $<0.001$ \\
\hline Inner-inferior & $53.10(4.19)$ & 44.17 (13.37) & $<0.001$ \\
\hline Outer-nasal & $39.43(3.64)$ & $33.41(7.19)$ & $<0.001$ \\
\hline Inner-nasal & $53.41(4.71)$ & $45.41(12.78)$ & $<0.001$ \\
\hline Foveal & $16.81(6.18)$ & $17.48(6.99)$ & 0.621 \\
\hline Volume & $1.12(0.09)$ & $0.93(0.22)$ & $<0.001$ \\
\hline \multicolumn{4}{|l|}{ IPL } \\
\hline Outer-superior & $29.75(2.81)$ & $25.07(4.21)$ & $<0.001$ \\
\hline Inner-superior & $41.65(2.89)$ & $36.53(7.96)$ & $<0.001$ \\
\hline Outer-temporal & $32.75(2.93)$ & $28.67(5.44)$ & $<0.001$ \\
\hline Inner-temporal & $40.50(3.45)$ & $36.90(9.00)$ & 0.018 \\
\hline Outer-inferior & $28.76(3.19)$ & $24.85(4.32)$ & $<0.001$ \\
\hline Inner-inferior & $41.26(2.98)$ & $36.63(7.65)$ & 0.001 \\
\hline Outer-nasal & $30.03(3.23)$ & $27.14(5.18)$ & 0.001 \\
\hline Inner-nasal & $42.65(3.36)$ & $38.22(7.63)$ & 0.001 \\
\hline Foveal & 21.20 & $21.80(20.67)$ & 0.495 \\
\hline Volume & $0.91(0.07)$ & $0.81(0.14)$ & $<0.001$ \\
\hline
\end{tabular}

${ }^{\mathrm{a}} t$-test 
Table 3 AUC and diagnostic sensitivity of thicknesses of the circumpapillary retinal nerve fiber layer, macular retinal nerve fiber layer, ganglion cell layer and inner plexiform layer

\begin{tabular}{|c|c|c|c|}
\hline & \multirow[t]{2}{*}{ AUC (SD) } & \multicolumn{2}{|l|}{ Sensitivity } \\
\hline & & Specificity fixed at $80 \%$ & Specificity fixed at $90 \%$ \\
\hline \multicolumn{4}{|l|}{ cpRNFL } \\
\hline Overall & $0.835(0.047)$ & 70.9 & 41.8 \\
\hline Sup-temporal & $0.869(0.038)$ & 69.1 & 61.8 \\
\hline Temporal & $0.746(0.057)$ & 38.2 & 12.7 \\
\hline Inf-temporal & $0.795(0.049)$ & 52.7 & 43.6 \\
\hline Inf-nasal & $0.777(0.051)$ & 49.1 & 21.8 \\
\hline Nasal & $0.724(0.056)$ & 43.6 & 20.0 \\
\hline Sup-nasal & $0.783(0.05)$ & 63.6 & 38.2 \\
\hline \multicolumn{4}{|l|}{ mRNFL } \\
\hline Outer-superior & $0.767(0.051)$ & 49.1 & 40.0 \\
\hline Inner-superior & $0.513(0.064)$ & 21.8 & 10.9 \\
\hline Outer-temporal & $0.601(0.06)$ & 43.6 & 27.3 \\
\hline Inner-temporal & $0.411(0.061)$ & 20.0 & 9.1 \\
\hline Outer- inferior & $0.697(0.06)$ & 36.4 & 1.8 \\
\hline Inner-inferior & $0.598(0.061)$ & 29.1 & 9.1 \\
\hline Outer-nasal & $0.649(0.059)$ & 38.2 & 23.6 \\
\hline Inner-nasal & $0.506(0.063)$ & 25.5 & 7.3 \\
\hline Foveal & $0.487(0.064)$ & 21.8 & 7.3 \\
\hline Volume & $0.672(0.052)$ & 34.5 & 14.5 \\
\hline \multicolumn{4}{|l|}{ CGL } \\
\hline Outer-superior & $0.840(0.044)$ & 80.0 & 54.5 \\
\hline Inner-superior & $0.692(0.057)$ & 47.3 & 23.6 \\
\hline Outer-temporal & $0.774(0.052)$ & 58.2 & 32.7 \\
\hline Inner-temporal & $0.625(0.063)$ & 23.6 & 14.5 \\
\hline Outer- inferior & $0.798(0.051)$ & 70.9 & 32.7 \\
\hline Inner-inferior & $0.693(0.059)$ & 50.9 & 23.6 \\
\hline Outer-nasal & $0.755(0.056)$ & 50.9 & 18.2 \\
\hline Inner-nasal & $0.657(0.06)$ & 40.0 & 25.5 \\
\hline Foveal & $0.445(0.064)$ & 14.5 & 5.5 \\
\hline Volume & $0.767(0.051)$ & 52.7 & 40.0 \\
\hline \multicolumn{4}{|l|}{ IPL } \\
\hline Outer-superior & $0.799(0.049)$ & 65.5 & 29.1 \\
\hline Inner-superior & $0.656(0.60)$ & 32.7 & 18.2 \\
\hline Outer-temporal & $0.714(0.057)$ & 52.7 & 9.1 \\
\hline Inner-temporal & $0.565(0.068)$ & 10.9 & 3.6 \\
\hline Outer- inferior & $0.742(0.054)$ & 47.3 & 30.9 \\
\hline Inner-inferior & $0.670(0.061)$ & 34.5 & 14.5 \\
\hline Outer-nasal & $0.694(0.06)$ & 41.8 & 12.7 \\
\hline Inner-nasal & $0.640(0.06)$ & 29.1 & 20.0 \\
\hline Foveal & $0.449(0.063)$ & 12.7 & 7.3 \\
\hline Volume & $0.707(0.058)$ & 45.5 & 18.2 \\
\hline
\end{tabular}

diagnostic capacity of inner macular layer segmentation analysis to differentiate between normal eyes and glaucomatous eyes, comparable to that of cpRNFL thickness, in adults [8].
To the best of our knowledge, this is the first study to compare the diagnostic performance of a Spectralis OCT macular segmentation software compared with a peripapillary analysis in children with PCG. Its findings indicate 


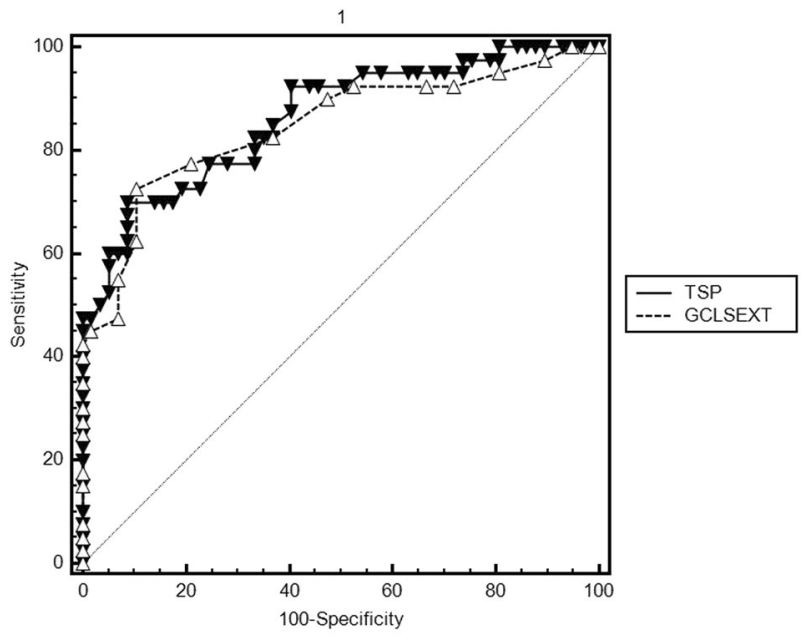

Fig. 2 Comparison between AUCs for the most discriminatory papillary variable (the cpRNFL superior temporal sector) and the best macular variable (outer-superior GCL sector)

reduced thicknesses in all sectors examined in the children with glaucoma compared to the control children both in our papillary and macular analysis. These observations are consistent with those of other studies. For instance, Ghasia et al [10] showed a decreased of cpRNFL thickness in pediatric glaucomatous eyes compared with healthy eyes and Silverstein et al. [12] reported significant statistical differences in volumes of each macular layers between healthy and glaucoma children using Spectralis OCT. We included foveal thickness corresponding to the $1 \mathrm{~mm}$ diameter central zone. As expected, no differences in this variable was detected between the normal and glaucomatous eyes in any of the three layers examined. The reason of the lack of differences in this central zone might be explained by the absence of all retinal layers except for the phothoreceptor layer which can be found isolated in this particular area.

The sectors showing the best diagnostic performance in our study were the outer-superior quadrant in all the macular layers examined and the superior temporal quadrant of the cpRNFL. Unlike this, published data for adults agree that the best macular and papillary variable for a diagnosis of glaucoma are those of the inferior temporal quadrant $[7,13,14]$. These results suggest that the topographic site of damage in children could differ from that produced in adults also reported in a pediatric PCG population a global loss of ganglion cell complex thickness and cpRNFL thickness that was particularly marked in the superior and inferior quadrants $[2,11]$.

While the diagnostic performance of the best macular variables in our study was similar to that of the best papillary variable, AUCs for all the circumpapillary factors were greater than those obtained for the macular factors. In contrast, Kim et al. [15]. observed in adults that
AUCs for overall mRNFL and GCL thicknesses (0.915 and 0.914$)$ tended to be greater than that for cpRNFL global thickness (0.878), and that the AUC for overall IPL thickness was lower. More recently, a similar study using the same new segmentation Software [8] found that the three best parameters were the temporal-inferior sector of pRNFL (AUC, 0.956), the outer inferior sector of mRNFL (AUC, 0.906), the outer-inferior sector of macular inner retinal layer (AUC, 0.876), the the inner-temporal of mGCL (AUC, 0.858) and the inner-temporal of IPL (AUC, 0.854). This study showed that pRNFL still performed better than the other retinal macular layers assessed separately. These differences could be explained because glaucomatous eyes included in the first study had moderate to severe disease, however most of the glaucomatous eyes included in the second one showed mild disease.

On the other hand, Martinez-de-la-Casa et al[13]. recorded a greater AUC for inner inferior mRNFL thickness than for the others sectors of GCL and IPL. In our study the discriminatory capacity of mRNFL thickness for all quadrants in children tended to be lower compared to GCL and IPL thicknesses.

This study reveals that segmented macular layer analysis shows a good capacity to discriminate between normal and glaucomatous eyes in children; which is comparable to that of cpRNFL analysis. However we observed important differences when we compare our results with other articles published previously in adults. We hypothesize that software of macular segmentation is more sensible to artifacts than papillary analysis in children. The thinner thickness of measurements for inner macular layers than cpRNFL, combined with poor fixation and poor cooperation in glaucoma children could generate lower reproducibility of inner macular layers measurements. Future studies should determine the repeatability and reproducibility of segmented macular layers measurements in children.

The main limitation of our study was the relatively small number of cases of PCG. Besides being fairly rare, it is difficult to recruit children with PCG because of opacity of the ocular media, poor fixation due to poor vision and a lack of cooperation at pediatric ages. All this creates a selection bias towards eyes with less severe clinical signs.

PCG is also characterized by axial lengthening [1] and as expected, we noted significant differences in axial length between groups. The axial length related magnification effect could interfere with both papillary and macular OCT measurements $[16,17]$.

To conclude, our results showed that segmented macular layer analysis has a good capacity to discriminate between normal and glaucomatous eyes; which is comparable to that 
of cpRNFL analysis in children with PCG. The main limitation of the use of this tool in children is that there is not normative database yet available. Despite this problem, this technique might be useful by comparing serial measurements over time.

\section{Summary}

\section{What was known before}

- Diagnosis of primary congenital glaucoma is based on clinical signs however the development of OCT allows to objective the structural damage.

- Utility of retinal nerve fiber layer measurements has been reported and good reproducibilty was observed in children.

\section{What this study adds}

- This study compares the diagnostic ability of two types of analysis using Spectral domain OCT in children with glaucoma: segmented ganglion cell complex and retinal nerve fiber layer analysis. There is no available information about the utility of the new macular segmentation software (usin Spectralis OCT) in children.

- This study reveals that diagnostic capacity of both analysis is good and comparable.

\section{Compliance with ethical standards}

Conflict of interest The authors declare that they have no conflict of interest.

\section{References}

1. Beck A, Chang TC, Freedman S. Definition, classification, differential diagnosis. In: Weinreb RN, Grajewski A, Papadopoulos M, Grigg J, Freedman S, editors. Childhood Glaucoma. Amsterdam: Kugler Publications; 2013. pp. 3-10.

2. Srinivasan S, Addepali UK, Rao HL, Garudadri CS, Mandal AK. Spectral domain optical coherence tomography in children operated for primary congenital glaucoma. $\mathrm{Br} \mathrm{J}$ Ophthalmol. 2014;98:162-5.

3. Ghasia FF, El-Dairi M, Freedman SF, Rajani A, Asrani S. Reproducibility of spectral-domain optical coherence tomography measurements in adult and pediatric glaucoma. J Glaucoma. 2015;24:55-63.

4. Vajzovic L, Hendrickson AE, O'Connell RV, et al. Maturation of the human fovea: correlation of spectral-domain optical coherence tomography findings with histology. Am J Ophthalmol. 2012;154:779-89.

5. Leung CK, Cheung CY, Weinreb RN, Qiu Q, Liu S, Li H, et al. Retinal nerve fiber layer imaging with spectral-domain optical coherence tomography: a variability and diagnostic performance study. Ophthalmology. 2009;116:1257-63.

6. Garcia-Martin E, Pinilla I, Idoipe M, Fuertes I, Pueyo V. Intra and interoperator reproducibility of retinal nerve fibre and macular thickness measurements using Cirrus Fourier-domain OCT. Acta Ophthalmol. 2011;89:e23-9.

7. Kim NR, Lee ES, Seong GJ, Kim JH, An HG, Kim CY. Structurefunction relationship and diagnostic value of macular ganglion cell complex measurement using Fourier-domain OCT in glaucoma. Invest Ophthalmol Vis Sci. 2010;51:4646-51.

8. Pazos M, Dyrda AA, Biarnés M, Gómez A, Martín C, Mora C, et al. Diagnostic Accuracy of Spectralis SD OCT Automated Macular Layers Segmentationto Discriminate Normal from Early Glaucomatous Eyes. Ophthalmology. 2017;124:1218-28.

9. Altemir I, Pueyo V, Elía N, Polo V, Larrosa JM, Oros D. Reproducibility of optical coherence tomography measurements in children. Am J Ophthalmol. 2013;155:171-6.

10. Ghasia FF, Freedman SF, Rajani A, Holgado S, Asrani S, El-Dairi M. Optical coherence tomography in paediatric glaucoma: time domain versus spectral domain. $\mathrm{Br} \quad \mathrm{J}$ Ophthalmol. 2013;97:837-42.

11. El-Dairi MA. Optical coherence tomography in the management of congenital glaucoma. Br J Ophthalmol. 2014;98:149-50.

12. Silverstein E, Freedman S, Zéhil GP, Jiramongkolchai K, El-Dairi $M$. The macula in pediatric glaucoma: quantifying the inner and outer layers via optical coherence tomography automatic segmentation. J AAPOS. 2016;20:332-6.

13. Martinez-de-la-Casa JM, Cifuentes-Canorea P, Berrozpe C, Sastre M, Polo V, Moreno-Montañes J, et al. Diagnostic ability of macular nerve fiber layer thickness using new segmentation software in glaucoma suspects. Invest Ophthalmol Vis Sci. 2014;55:8343-8.

14. Kotera Y, Hangai M, Hirose F, Mori S, Yoshimura N. Threedimensional imaging of macular inner structures in glaucoma by using spectral-domain optical coherence tomography. Invest Ophthalmol Vis Sci. 2011;52:1412-21.

15. Kim HJ, Lee SY, Park KH, et al. Glaucoma diagnostic ability of layer-by-layer segmented ganglion cell complex by spectraldomain optical coherence tomography. Invest Ophthalmol Vis Sci. 2016;57:4799-805.

16. Nakanishi H, Akagi T, Hangai M, Kimura Y, Suda K, Hasegawa $\mathrm{T}$, et al. Effect of axial length on Macular Ganglion Cell Complex Thickness and on early glaucoma diagnosis by spectral-domain optical coherence tomography. J Glaucoma. 2016;25:e481-90.

17. Wang XY, Huynh SC, Burlutsky G, Ip J, Stapleton F, Mitchell P. Reproducibility of and effect of magnification on optical coherence tomography measurements in children. Am J Ophthalmol. 2007;143:484-8. 